\title{
Quilotórax bilateral en un caso de adenocarcinoma metastásico de primario desconocido
}

\author{
A. GUTIÉRREZ MACÍAS, E. LIZARRALDE PALACIOS, J. M. MERINO MÚGICA, \\ S. CABEZA GARCÍA, P. MARTÍNEZ ODRIOZOLA, F. MIGUEL DE LA VILLA \\ Servicios de Medicina Interna y Respiratorio. Hospital de Basurto. Bilbao
}

\begin{abstract}
BILATERAL CHYLOTHORAX IN A CASE OF METASTATIC ADENOCARCINOMA OF UNKNOWN PRIMARY
\end{abstract}

\begin{abstract}
RESUMEN
Presentamos el caso de una paciente diagnosticada de quilotórax bilateral y de linfangitis carcinomatosa pulmonar, que evolucionó de forma desfavorable conduciendo al fallecimiento por insuficiencia respiratoria, en el contexto de un adenocarcinoma metastásico de primario desconocido tras el estudio necrópsico. A partir de este caso, revisamos la etiología, el diagnóstico y las opciones disponibles en el tratamiento de los quilotórax.
\end{abstract}

PALABRAS CLAVE: Quilotórax bilateral. Adenocarcinoma de primario desconocido.

\begin{abstract}
We present a case of bilateral chylothorax and lung carcinomatous lymphangitis. Clinical evolution was unfavorable, leading to death due to respiratory insufficiency. Necropsy showed widespread metastatic adenocarcinoma of unknown primary. From this case, we review the etiology, diagnosis and therapeutic options available in chylothorax.
\end{abstract}

KEY WORDS: Bilateral chylothorax. Adenocarcinoma of unknown primary.

Gutiérrez Macías A, Lizarralde Palacios E, Merino Múgica JM, Cabeza García S, Martínez Odriozola P, Miguel de la Villa F. Quilotórax bilateral en un caso de adenocarcinoma metastásico de primario desconocido. An Med Interna (Madrid) 2006; 23: 176-178.

\section{INTRODUCCIÓN}

El quilotórax es un trastorno poco frecuente, caracterizado por la acumulación de quilo en el espacio pleural, asociada a lesiones por rotura, compresión extrínseca u obliteración del conducto torácico o alguna de sus divisiones principales (1-4). Las causas de los quilotórax pueden agruparse en neoplasias, traumatismos, idiopáticos y miscelánea. Las neoplasias son la causa más frecuente; entre estas destacan los linfomas (sobre todo no Hodgkin) que constituyen prácticamente un $50 \%$ del total de los casos (5). Aunque es una eventualidad más rara, otras neoplasias no linfoides pueden producir un quilotórax. Presentamos un caso de adenocarcinoma metastásico sin primario identificado en la necropsia, cuyas primeras manifestaciones clínicas se derivaron de la existencia de una linfangitis carcinomatosa y un quilotórax bilateral.

\section{CASO APORTADO}

Mujer de 73 años con antecedentes de diabetes mellitus tipo 2 en tratamiento con antidiabéticos orales, cardiopatía isquémica, hiper- tensión arterial y fibrilación auricular paroxística en tratamiento con amiodarona, calcioantagonistas y antiagregantes plaquetarios. Refería un cuadro de 2 meses de evolución de tos, fiebre y disnea. En las pruebas complementarias destacaban anemia normocítica, hipoxemia, elevación de reactantes de fase aguda e infiltrados "parcheados" en ambos hemitórax con derrame pleural derecho. Se realizaron múltiples estudios microbiológicos, serológicos, citológicos y de imagen que fueron negativos o normales. Se estableció el diagnóstico de presunción bronquiolitis obliterante con neumonía organizada (BONO) y se inició tratamiento con prednisona $60 \mathrm{mg}$ /día con desaparición de la fiebre y mejoría de los síntomas respiratorios, de la anemia y de los reactantes de fase aguda. La paciente fue ingresada de nuevo un mes más tarde por disnea de reposo, edemas y oliguria. En la exploración destacaban taquipnea, taquicardia rítmica sin soplos, disminución global del murmullo vesicular con crepitantes en bases y edemas en extremidades inferiores. Entre las pruebas de laboratorio destacaban 20.600 leucocitos $/ \mathrm{mm}^{3}$, con $85 \%$ polimorfonucleares, LDH $537 \mathrm{U} / \mathrm{L}$, fosfatasa alcalina $249 \mathrm{U} / \mathrm{L}$, albúmina 2,3 g/dL, $\mathrm{pO}_{2} 58$ mmHg y CA 19.9214 U/L (normal 0-27 U/L). Eran normales las cifras de hemoglobina, plaquetas, glucemia, bilirrubina, función renal, calcio, electrolitos, antígeno carcinoembrionario, CA 125 y determinación de hormonas tiroideas. En la radiografía de tórax y la tomografía computarizada (TC) torácica se observaban derrame pleural bilateral y un aumento de la densidad bilateral en el parénqui-

Trabajo aceptado: 28 de octubre de 2005

Correspondencia: A. Gutiérrez Macías. Servicio de Medicina Interna. Hospital de Basurto. Avda. de Montevideo, 18. 48013 Bilbao. e-mail: alguna@teleline.es 


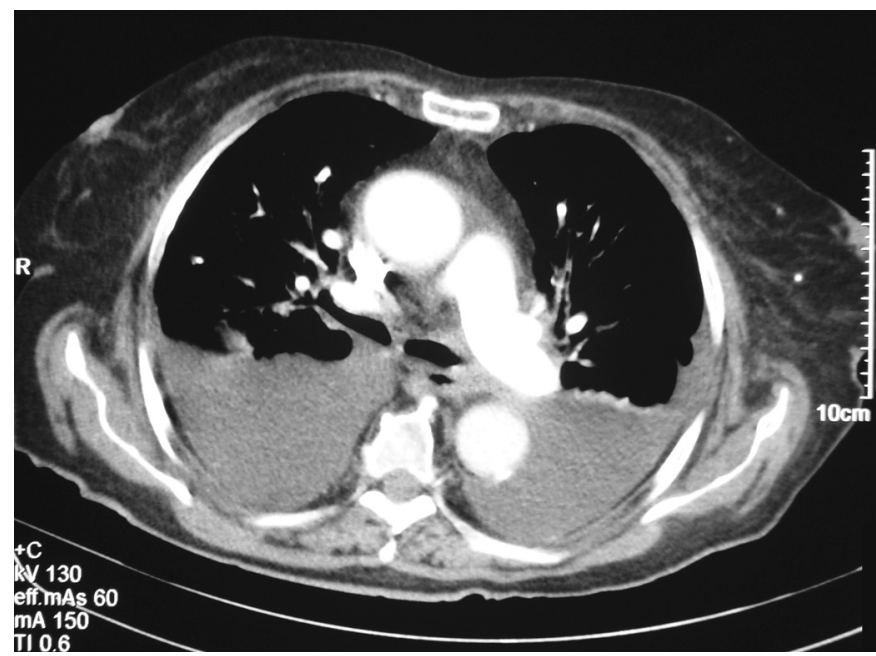

Fig. 1. Tomografía computarizada con derrame pleural bilateral y aumento de densidad del parénquima pulmonar de predominio perihiliar.

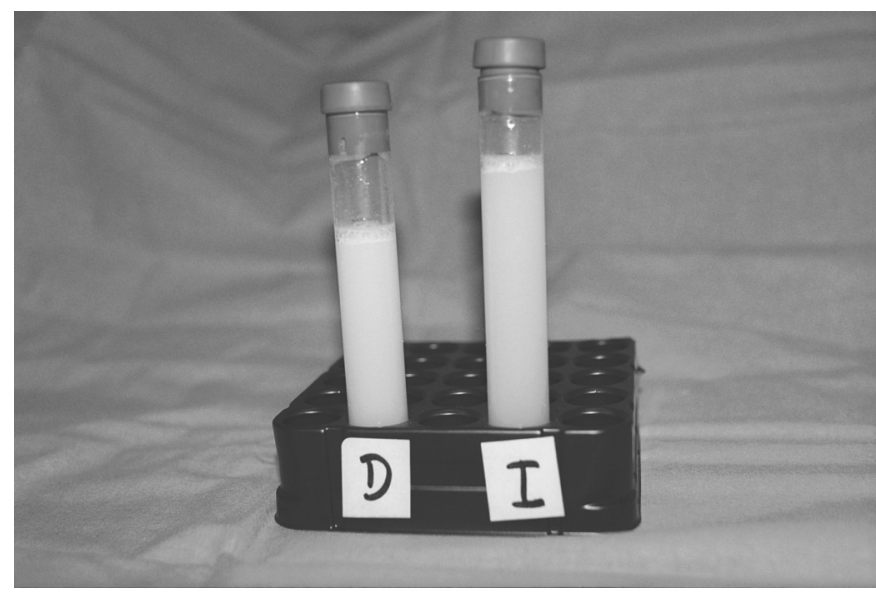

Fig. 2. Aspecto de los líquidos pleurales obtenidos por toracocentesis. D: derecho. I: izquierdo.

ma pulmonar, de predominio perihiliar (Fig. 1). El mediastino era normal y no había signos de embolismo pulmonar en la angio-TC. Un ecocardiograma transtorácico y una TC abdominal fueron normales. Se realizó toracocentesis bilateral obteniendo en ambos casos un líquido pleural de aspecto lechoso (Fig. 2), con características de exudado, concentración de triglicéridos de $433 \mathrm{mg} / \mathrm{dL}$ y $163 \mathrm{mg} / \mathrm{dL}$ respectivamente, y presencia de quilomicrones (Tabla I). Los estudios citológicos y microbiológicos de los líquidos pleurales fueron negativos. Se realizó una broncoscopia con biopsia transbronquial en la que se observaba una mucosa bronquial infiltrada por una proliferación neoplásica de células de adenocarcinoma. Las citologías y estudios microbiológicos del broncoaspirado y del lavado broncoalveolar fueron negativos. Se incrementó la dosis de esteroides y se realizó tratamiento con tubo de drenaje torácico bilateral y nutrición parenteral. La evolución posterior se caracterizó por una insuficiencia respiratoria progresiva que condujo al fallecimiento de la paciente. En la necropsia se observó una linfangitis carcinomatosa diseminada con afectación de pulmones, hígado, pericardio, páncreas y suprarrenales, secundaria a adenocarcinoma de primario desconocido.
TABLA I

VALORES DE LABORATORIO DE LOS LÍQUIDOS PLEURALES

\begin{tabular}{lcc}
\hline & Derecho & Izquierdo \\
\hline Aspecto & Lechoso & Lechoso \\
Proteínas totales $(\mathrm{g} / \mathrm{dL})$ & 3,42 & 3,48 \\
LDH $(\mathrm{U} / \mathrm{L})$ & 248 & 225 \\
Leucocitos $\left(/ \mathrm{mm}^{3}\right)$ & 740 & 990 \\
Linfocitos $(\%)$ & 60 & 45 \\
Colesterol $(\mathrm{mg} / \mathrm{dL})$ & 143 & 126 \\
Triglicéridos $(\mathrm{mg} / \mathrm{dL})$ & 433 & 163 \\
Quilomicrones & + & + \\
ADA $(\mathrm{U} / \mathrm{L})$ & 23 & 23 \\
\hline
\end{tabular}

$\mathrm{LDH}$ : láctico deshidrogenasa. ADA: adenosina desaminasa.

\section{DISCUSIÓN}

El quilotórax se define como la acumulación de quilo en el espacio pleural, asociada a lesiones que afectan al conducto torácico o alguna de sus divisiones principales (1-4). Otros mecanismos de formación del quilotórax, como la extravasación a partir de los vasos linfáticos pleurales o el flujo transdiafragmático de una ascitis quilosa, son menos importantes (4). El aspecto lechoso característico del quilotórax se debe a la presencia de quilomicrones; debe distinguirse del pseudoquilotórax, en el que el este aspecto se debe al alto contenido en colesterol en derrames de evolución crónica $(1,2)$. El quilotórax es un trastorno poco frecuente; se estima que puede aparecer en el 3\% de los derrames pleurales que requieren toracocentesis en adultos (6). La pérdida de quilo tiene como consecuencias la depleción de líquidos, electrolitos, proteínas, grasas, vitaminas liposolubles y linfocitos (principalmente T), que condiciona una alteración de gravedad variable del estado nutricional y de la inmunidad humoral y celular $(2,7)$. Por otra parte, la acumulación de quilo en el espacio pleural produce un compromiso en la ventilación y en la función cardiaca, derivados de la existencia de fenómenos compresivos locales (7).

La etiología de los quilotórax en adultos puede dividirse en cuatro categorías: neoplasias, traumatismos, idiopáticos y causas misceláneas (4). Las neoplasias son la causa más frecuente; entre estas destacan los linfomas (sobre todo no Hodgkin) que constituyen prácticamente un $50 \%$ del total de los casos (5). La existencia de un quilotórax como complicación de neoplasias no linfoides, como en el caso que presentamos, es una circunstancia mucho menos frecuente (2). Los traumatismos son la segunda causa en orden de frecuencia, alcanzando un $25 \%$ aproximadamente; generalmente están en relación con intervenciones quirúrgicas sobre la región cervical o la cavidad torácica, o con la cateterización de vasos venosos de gran calibre para nutrición parenteral o monitorización hemodinámica. En algunos casos pueden observarse quilotórax tras traumatismos torácicos o abdominales (abiertos o cerrados), vómitos o accesos de tos. Entre un 10-15\% de los casos de quilotórax son idiopáticos; se supone que pueden estar relacionados con traumatismos banales, tras la ingesta de comidas ricas en grasas (3). Las causas misceláneas (8-10\%) pueden incluir linfangioleiomiomatosis, esclerosis tuberosa, tumores 
benignos, aneurismas, tuberculosis, sarcoidosis, amiloidosis, lupus eritematoso, hemangiomatosis (síndrome de Gorham), enfermedad de Castleman, bocio retroesternal, síndrome de cava superior, trombosis de la subclavia o insuficiencia cardiaca $(3,4,8)$. Aunque clásicamente se ha considerado que la cirrosis era una causa excepcional de quilotórax, en alguna serie es la segunda etiología después de las neoplasias (6); en estos casos, suele asociarse a ascitis quilosa y el líquido presenta características bioquímicas de trasudado (3).

El diagnóstico definitivo de quilotórax exige la demostración de quilomicrones en el líquido pleural (2,3); sin embargo, se admite que un líquido con una concentración de triglicéridos $>110 \mathrm{mg} / \mathrm{dL}$ tiene una alta probabilidad de corresponder a un quilotórax (9), sobre todo si coexisten un cociente de colesterol líquido pleural/suero menor de 1 y un cociente de triglicéridos líquido pleural/suero mayor de 1, que descartan el pseudoquilotórax y la hipertrigliceridemia grave respectivamente (3).

El tratamiento conservador del quilotórax incluye el drenaje torácico con toracocentesis repetidas o con tubo de tórax y la nutrición parenteral para mantener el estado nutricional y disminuir el flujo de quilo a través del conducto torácico $(2,7)$. En los casos de quilotórax que no se asocien a un compromiso cardiorrespiratorio, puede ser suficiente la observación clínica, mientras se inicia el tratamiento de la causa subyacente (4). En cuanto a las medidas nutricionales, los triglicéridos de cadena media (TCM) por vía oral son absorbidos directamente por el sistema portal, disminuyendo el flujo de quilo por el conducto torácico; sin embargo, dado que cualquier ingesta oral puede aumentar el flujo linfático y que no todos los TCM son absorbidos por esta vía, actualmente se recomienda la dieta absoluta con nutrición parenteral total (3). Aunque la experiencia en su utilización es aún muy limitada, la administración de somatostatina o sus análogos (octreótido), puede constituir un tratamiento coadyuvante eficaz y seguro $(10,11)$. Su mecanismo de acción no se conoce por completo, aunque podría estar relacionado con la vasoconstricción que ejercen sobre los linfáticos esplácnicos (10). El tratamiento quirúrgico está indicado cuando fracasa el tratamiento conservador; el procedimiento de elección es la ligadura del conducto torácico inmediatamente por encima del diafragma derecho, que puede hacerse con toracotomía o videotoracoscopia (con menor morbilidad). El momento de la cirugía es un tema controvertido; generalmente está indicada si existe un deterioro nutricional evidente, cuando el drenaje es $>1.500 \mathrm{~mL} /$ día o cuando no existe mejoría tras dos semanas de tratamiento conservador $(1,3,6)$. En algunos casos se han realizado derivaciones pleuroperitoneales o pleurodesis con talco o tetraciclinas con resultados variables $(4,7)$. Además de las medidas indicadas, debe realizarse tratamiento etiológico en los casos en que sea posible (quimio y radioterapia en los linfomas).

\section{Bibliografía}

1. Sassoon CS, Light RW. Chylothorax and pseudochylothorax. Clin Chest Med 1985; 6: 163-71.

2. de Beer HGJ, Mol MJT, Janssen JP. Chylothorax. Neth J Med 2000; 56: 25-31.

3. Romero S. Nontraumatic chylothorax. Curr Opin Pulmonary Med 2000; 6: 287-91.

4. Doerr CH, Miller DL, Ryu JH. Chylothorax. Sem Respir Crit Care Med 2001; 22: 617-26.

5. Valentine VG, Taffin TA. The management of chylothorax. Chest 1992; 102: 586-91.

6. Romero S, Martín C, Hernández L, Verdú CJ, Trigo C, Pérez-Mateo M, et al. Chylothorax in cirrosis of the liver: análisis of its frequency and clinical characteristics. Chest 1998; 114: 154-9.

7. Merrigan BA, Winter DC, O'Sullivan GC. Chylothorax. Br J Surg 1997; 84: $15-20$.

8. Saumoy M, Mirón M, Oltra C, Vidal F, Richart C. Quilotórax tuberculoso: caso clínico y revisión de la literatura. An Med Interna (Madrid) 2005; 22: 238-40.

9. Staats BA, Ellefsonn RD, Budahn LL, Dines DE, Prakash USB, Oxford $\mathrm{K}$. The lipoprotein profile of chylous and nonchylous pleural effusions. Mayo Clin Proc 1980; 55: 700-4.

10. Buettiker V, Hug MI, Burger R, Baenziger O. Somatostatin: a new therapeutic option for the treatment of chylothorax. Intensive Care Med 2001; 27: 1083-6.

11. Demos NJ, Kozel J, Scerbo JE. Somatostatin in the treatment of chylothorax. Chest 2001; 119: 964-6. 